\title{
Cellular mysteries of plant sex
}

\author{
Peter Nick $^{1}$
}

Received: 19 September 2016 / Accepted: 19 September 2016/Published online: 28 September 2016

(C) Springer-Verlag Wien 2016

Whether plant sexuality exists at all, had been disputed over centuries. While so evident in animals, in plants, even subtle symptoms of mating behaviour are not detected. Moreover, with a few exceptions, plants are not "male" or "female". It was only in 1759, when Joseph Kölreuter in St. Peterburg could demonstrate the existence of plant sexuality at all by a symmetric cross between two morphologically different tobacco species. During these experiments that had been conducted in response to one of the first science awards published by Tsar Catharina II to resolve the dispute about plant sex, Kölreuter arrived at the conclusion that both parents equally contribute to inheritance which implicated two versions for each trait, one inherited from the father, and the second inherited from the mother. A century later, this finding by Kölreuter helped Gregor Mendel to conceive the laws of dominant-recessive inheritance, explaining the long-lasting mystery of so called latency, where a trait that was not manifested in the parents, but nevertheless could be inherited to the next generation.

Over the decades, the science of inheritance, later called genetics, developed rules of progressive complexity to describe the frequency of individual traits or their combinations in a crossing progeny. This approach remained purely mathematical, because the material base of inheritance became accessible only from the 1950s. An implicit assumption of this mathematical branch of biology was the assumption that sexuality is symmetrical, because, during fertilisation, two corresponding chromosome sets are merged, and later, during meiosis, this double set is redistributed into two corresponding

Peter Nick

peter.nick@kit.edu

1 Botanical Institute, Karlsruhe Institute of Technology, Karlsruhe, Germany chromosome sets again. This concept of symmetrical sex was challenged, when it became clear that also mitochondria and plastids contribute to inheritance and that this inheritance is often only matrilinear. However, these deviations were mostly considered as variations to the theme of sexual symmetry.

It seems that, at least for plants, the cellular details of sexuality still are good for surprises, and these surprises are highly relevant for the way, how we think about inheritance. Two contributions in the current issue reveal new and astonishing details of plant sex that are of great relevance for genetics:

Diatoms have fascinated generations of biologists by the beauty of their symmetrical shells. However, they also have been fascinating as one of the few cases of a micro-organism with a diploid life style. Since the shell is composed of a larger lid covering a smaller base, these cells will become smaller and smaller during mitotic divisions. Sexuality allows to reestablish a non-walled auxospore, which can then generate a new and large founder cell for a new lineage of mitotic divisions. This sexual step begins, when two cells of opposite mating type align and transform into gametangia. Each gametangium will undergo meiosis giving rise to two gametes. The two gametes from the "male" gametangium will move towards their "female" counterparts and conjugate. The zygote will then convert into an auxospore. In careful time course experiments, Scalco et al. (2016), in the current issue, followed this process by confocal laser scanning microscopy after fluorescent visualisation of the nucleus in the model species Pseudonitzschia striata and thus were able to uncover so far unknown details of the conjugation process that bear consequences for the molecular genetics of this organism. In particular, for the first time, detailed time courses of the different steps could be established. When authors followed meiosis and subsequent conjugation, they discovered a complex and sophisticated behaviour of chloroplasts: The two chloroplasts of the gametangial cell divide and the resulting 
daughters of each chloroplast move apart towards the cell poles. As a consequence, each gamete will get a mixed couple of daughter plastids. In other words - unlike in most higher plants, plastidic inheritance in this diatom is symmetric, which means that plastidic genes are inherited through both, the maternal and the paternal line, a mechanism that is suited to increase genetic diversity.

The contribution by Mursalimov et al. (2016) in the current issue deals with the curious phenomenon of cytomixis, where during microsporogenesis, entire nuclei move through specific intracellular channels from cell to cell. While this phenomenon had been described already at the beginning of the last century and seems to occur in numerous plant species, the underlying mechanisms and driving forces have remained obscure, which is partially caused by the fact that the frequency of cytomixis strongly depends on unknown factors that varied between experiments from different laboratories. In their previous work (Mursalimov and Deineko 2011), the authors have characterised the cellular and ultrastructural aspects of cytomixis in tobacco microsporogenesis. In their current contribution, they have now systematically addressed the impact of ploidy levels upon the frequency of cytomixis. The idea of this experiment is as simple as elegant: By means of classical colchicine treatments, they have generated a series of tobacco plants that were isogenic, but differed in their ploidy levels ranging from 24 over to 96 chromosomes. These plants were now raised side by side under exactly the same conditions to filter out any environmental factors that might modulate cytomixis. Then they analysed microsporogenesis microscopically and quantified the incidence of cytomictic events. They arrive at two important conclusions: First, the rate of cytomixis increases with ploidy level, and second, even under their stringent regime, the frequency of cytomixis remained variable (which indicates that the activation of cytomixis is a self-amplifying process that is therefore susceptible to even subtle details of cultivation, hormonal regimes or even circadian rhythm). Since cytomixis is observed in hundreds of plant species, it is of evolutionary relevance. The authors discuss whether cytomixis might be a mechanism to get rid of surplus chromosomes in polyploids as mechanism to reestablish genetic integrity, but argue against this "rescue" function, because it accompanies numerous additional meiotic abnormalities. However, they emphasise that cytomixis occurs to a certain rate even in normal diploids and generates binucleate microsporocytes during the first meiotic prophase. Although the frequency of this event is low (around $1 \%$ ), it is not negligible and might be one of the cellular factors facilitating allopolyploid as one of the most powerful driving forces for plant speciation.

Both contributions to cellular aspects of plant sex demonstrate two points: Even more than 120 years after Hertwig's first full description of meiosis, still many cellular details are unknown. There seem to be numerous variations to the theme and these variations are genetically relevant. Without a deeper understanding of the cellular mysteries of meiosis we will not be able to arrive at a real understanding of evolution.

\section{Compliance with ethical standards}

Conflict of interest The author declares that there is no conflict of interest.

\section{References}

Mursalimov SR, Deineko EV (2011) An ultrastructural study of cytomixis in tobacco pollen mother cells. Protoplasma 248:717-724

Mursalimov SR, Sidorchuk Y, Demidov D, Meister A, Deineko E (2016) A rise of ploidy level influences the rate of cytomixis in tobacco male meiosis. Protoplasma, current issue

Scalco E, Amato A, Ferrante MI, Montresor M (2016) The sexual phase of the diatom Pseudonitzschia multistriata: cytological and timelapse cinematography characterization. Protoplasma, current issue 